\title{
SIGNIFICADO ESTRUCTURAL Y EXTENSIÓN SEMÁNTICA EN LOS COMPUESTOS ESPAÑOLES CON VERBOS
}

\author{
Bárbara Marqueta Gracia \\ Universidad de Zaragoza
}

\section{RESUMEN}

En la presente propuesta se presentan de manera sucinta las principales diferencias semánticas entre algunos esquemas compositivos españoles equicategoriales -integrados por constituyentes de las mismas categorías- $(\mathrm{N}+\mathrm{N}, \operatorname{Adj}+\mathrm{N}, \mathrm{V}+\mathrm{N} / \mathrm{N}+\mathrm{V})$. Las diferencias semánticas se localizan, esencialmente, en dos niveles de análisis: la relación entre denotación y referente, tanto de los constituyentes del compuesto como de los compuestos enteros, y las relaciones semánticas entre constituyentes. Respecto al primer nivel, veremos que la clave para explicar los contrastes reside en la estructura argumental del compuesto, que es mucho más rígida en el caso del verbonominal, aunque también localizaremos diferencias más sutiles que dependen de la asignación de roles temáticos. Respecto al segundo nivel, los compuestos llamados "de núcleo a la derecha», como vasodilatador, presentan una pauta común que hace legítimas diversas relaciones entre sus constituyentes (las conocidas como argumentales, pero también las adjuntas, como radiodiagnóstico). Los compuestos «de núcleo a la izquierda», sin embargo, presentan una relación semántica única en sus esquemas, siendo la denotación de los constituyentes sustantivos la que permitirá distinguir entre ciertos matices semánticos distintivos en estos compuestos.

Palabras Clave: compuesto, argumento, rol temático, sustantivo, verbo.

\section{STRUCTURAL MEANING AND SEMANTIC EXTENSION IN SPANISH VERBAL COMPOUNDS}

\section{Abstract}

This paper introduces the topic of semantic distinctions between Spanish compound patterns (e.g., $\mathrm{N}+\mathrm{N}, A d j+N)$. The semantic differences will be identified according to two parameters: the relationship between the extralinguistic entities and the compounds or their parts of them, and the semantic relationships inside the compounds. On the first topic, the argument structure of the compounds will be the key of the distinction, since the structure of $\mathrm{V}+\mathrm{N}$ compounds is unchanging, whereas the one of $\mathrm{N}+\mathrm{V}$ compounds is variable. More fine-grained differences will be identified from the point of view of thematic role assignment. On the second topic, the so-called «right-headed compounds» share a wide range of alternating semantic relationships, but the "left-headed compounds» show a unique semantic relationship. On the other hand, they show some distinctive features depending on the kind of noun classes that appear as their non-heads.

KEYWORDS: compound, argument, thematic role, noun, verb.

DOI: https://doi.org/10.25145/j.refiull.2020.40.09

Revista de Filología, 40; enero 2020, pp. 167-179; ISSN: e-2530-8548 


\section{INTRODUCCIÓN}

El estudio del significado en el ámbito de la morfología constituye uno de los más prolíficos y controvertidos. Prueba de ello es que no hay siquiera consenso respecto a cuál es el objeto de estudio; así, desde hace décadas, coexisten modelos «basados en el morfema», que manejan unidades morfológicas dotadas de significado (Plag 1999), y aquellos «basados en la palabra», que mantienen la centralidad del lexema como unidad significativa (Stump 2001). A ellos se añaden los modelos que asumen la "Hipótesis de la separación» (Beard 1995), en los que cualquier unidad morfológica es una correspondencia no necesariamente unívoca entre ciertos rasgos y su expresión - por lo que encontramos múltiples sufijos (saltador, gobernante, cocinero) que derivan "agentes" $\mathrm{y}$, a su vez, cada uno de ellos se corresponde con otros significados, como el de «lugar» (recibidor, pendiente, ropero)-.

El debate en torno a la unidad mínima significativa se acompaña de otras controversias, como, por ejemplo, qué tipo de significado aportan las unidades morfológicas, cómo dicho significado se codifica, en qué componente gramatical lo hace y la cuestión que es, en mi opinión, la de mayor relevancia para el estudio de los compuestos, que abordamos en la presente contribución: cómo integrar en el análisis del significado los aspectos «sistemáticos»o «composicionales»-aquellos que me permiten, por ejemplo, relacionar lavavajillas con un tipo deducible de actividad- y los aspectos «arbitrarios» 0 «impredecibles»-que hacen infructífero todo intento de deducir a qué se refiere pasodoble a partir del significado de las palabras paso y doble-. Autores como Lieber (2004), Pustejovsky (1995) o Ackema y Neeleman (2004), entre otros, plantean modelos de diferentes características para abordar dichas cuestiones.

Los estudios sobre la semántica de los compuestos se centran principalmente en dos aspectos. El primero de ellos es la relación entre el compuesto y la realidad lingüística a la que hace referencia, y el segundo es las relaciones semánticas entre constituyentes.

En el caso de la relación entre compuestos y referentes, destaca su protagonismo en la obra de Bustos (1986), aunque más recientemente encontramos propuestas como la de Buenafuentes (2017), quien aborda el asunto desde el punto de vista de la Semántica Cognitiva. Como señalan ambos autores, el grueso de la composición en español es de naturaleza nominal, por lo que la composición da lugar a sustantivos como contraventana, una unidad que tiene como referente una realidad extralingüística particular diferente, aunque relacionada en este caso con la de ventana ${ }^{1}$. El aspecto más prolífico de esta dimensión semántica de la composición es, sin duda, su relación con la identificación del núcleo de los compuestos, que es una cuestión particularmente compleja, puesto que se aborda no solo desde una perspec-

Cabe matizar que entendemos designación como la relación entre una unidad lingüística y un objeto de la realidad extralingüística al que pone nombre, y por referencia a la manifestación lingüística concreta de dicha relación en una situación comunicativa concreta. 
tiva semántica, sino también desde una perspectiva morfosintáctica (Scalise, Fábregas y Forza 2009). Así, una ventana y una contraventana se relacionan con referentes distintos, pero el significado de la segunda parece relacionarse esencialmente con el de la primera (qué entendemos por contraventana requiere de nuestro conocimiento de lo que es una ventana, a efectos de su contraste), y además, también parece que la adscripción al género femenino de contraventana está motivada por el hecho de que ventana posea el mismo género, y así sucede igualmente, como norma general, en construcciones como sofá cama, donde el género (masculino) es común al compuesto y al sustantivo identificado como núcleo, el sofá. Pese a ello, contraventana designa más bien un tipo de puerta que de ventana. Este aspecto es problemático desde la perspectiva adoptada por autores como Rainer y Varela (1992), para los cuales el núcleo del compuesto debe ser un concepto hiperónimo del compuesto; es decir, que contraventana refiera a un tipo de ventana sería una condición sine qua non para considerar la construcción endocéntrica (con núcleo ventana), mientras que para autores como Bauer (2008) no lo es.

No somos partidarios de la relevancia gramatical de la identificación de núcleos semánticos -en un mismo patrón compositivo coexisten compuestos que lo cumplen, como contrapuerta, que es una puerta, con compuestos que lo incumplen, como contraveneno, que no es un veneno, sino un antídoto-. Ello no implica en modo alguno que la relación entre los compuestos y sus posibles referentes sea irrelevante. Por ello, en este trabajo abordaremos dicho asunto de manera independiente del asunto de la nuclearidad, y lo haremos comparando las propiedades de los compuestos con verbos desde el punto de vista de su potencial denotativo, que definimos como la relación entre las propiedades de los verbos que aparecen en el compuesto y el tipo de referentes que denota este último. Veremos que, gracias a este criterio, podemos identificar diferencias sistemáticas significativas entre patrones compositivos.

El grueso de los estudios sobre la semántica de los compuestos en la tradición anglosajona dedica una mayor atención a las relaciones semánticas entre los constituyentes. Las propuestas oscilan de acuerdo al mayor o menor protagonismo que conceden a las reglas o al contexto/frecuencia de uso a la hora de explicar las relaciones identificadas (Lees 1960, Downing 1977, Gagné y Spalding 2006), y también difieren en la manera en que formalizan dichas relaciones (Lieber 2016, Jackendoff 2016). El análisis de la relación semántica entre constituyentes acarrea preguntas, por ejemplo: a la hora de interpretar tree house como 'casa en un árbol' y no como 'casa hecha de madera' o 'casa con forma de árbol', ¿̇se elige 'casa en un árbol' por ser la única interpretación semántica asociada a ese compuesto en particular, la más adecuada entre múltiples opciones, o acaso la más productiva para esa clase de compuestos? ¿Existe la misma disponibilidad de lecturas semánticas en todas las lenguas? ¿Y para todas las clases de compuestos?

Nuestra hipótesis es que la disponibilidad de relaciones semánticas es diferente en función tanto de la lengua como de la clase de compuesto analizada. Dado que nuestro análisis se centra en los compuestos verbales del espańol, haremos únicamente una breve presentación de estas diferencias en los compuestos de los tipos no verbales $\mathrm{N}+\mathrm{N}$ y $\mathrm{N}+\mathrm{Adj}$. 
En los compuestos $\mathrm{N}+\mathrm{N}$, el espańol presenta dos esquemas constructivos con evidentes diferencias morfofonológicas entre sí -respecto al número de acentos o la flexión nominal-: los compuestos apositivos de (1) y los compuestos léxicos de (2). La pregunta es ¿¿difieren también desde el punto de vista de sus relaciones semánticas?

1. perro policía, ley mordaza, madre coraje, guerra relámpago

2. papamóvil, radiodiagnóstico, hidroavión

Observamos que en el caso de (1) los sustantivos apuestos (policía, salchicha, relámpago...) pertenecen a clases conceptuales diversas (oficios como policía, cualidades como coraje, instrumentos como mordaza); dicha diversidad determina en gran medida, bien la subclasificación que se va a hacer del núcleo - p. e., una subclase de perro distintiva por su profesión (policía) o forma (salchicha)-, bien la adscripción de una propiedad al mismo (guerra súbita como un relámpago).

En el esquema de (2), sin embargo, la contribución semántica de los modificadores a los sustantivos nucleares, móvil, diagnóstico y avión, es más bien la de un adjetivo de los llamados 'relacionales': el papamóvil es el vehículo que transporta al papa, radio introduce el método por el que se efectúa el diagnóstico e hidro el lugar por el que el avión se desplaza. Es decir, la relación semántica entre constituyentes demanda la mediación, en el caso de (2), de alguno de los habituales roles temáticos de argumentos y adjuntos (agente, tema, instrumento, locación), lo que no sucede en (1), donde la interpretación semántica depende únicamente de las propiedades conceptuales de los sustantivos no núcleos (p. e., la profesión policía, lo «súbito» del relámpago).

En los compuestos $\mathrm{N}+\mathrm{Adj}$, observamos otro claro contraste entre la pauta establecida por el patrón compositivo de pelirrojo, cuya característica morfofonológica distintiva en español es el cierre en $-i$ del sustantivo (3), y otros compuestos adjetivos que no presentan necesariamente dicha vocal (4).

\section{3. patilargo, boquisucio, capipardo}

koridoustau 'corazoniduro'; pruppidruttfi 'pulpidulce' maniyilongu 'manillilargo' (Pinto et al., 2012: 54-56)

4. fotoalérgica (a las instantáneas y no a la luz), acidorresistente, beatlemaniaca

En este caso, los compuestos contrastan, no tanto desde el punto de vista de las relaciones entre constituyentes, sino desde el punto de vista de la relación entre el sustantivo ubicado en el interior del compuesto - pat(a), cap (a), foto, ácido...-y aquel al que se le atribuye el adjetivo compuesto en su totalidad -la persona con patas largas, la cosa resistente al ácido, etc.)-.

Como ha sido ampliamente destacado por la bibliografía (Gil Laforga 2014), los sustantivos de la pauta de (3) denotan exclusivamente partes constitutivas o «inalienables» del sustantivo al que se atribuye la cualidad (patas, boca), aunque qué clases de sustantivos se admiten como inalienables puede variar entre construcción y construcción; el compuesto español representado por pelirrojo, por ejemplo, manifiesta 
preferencia por partes del cuerpo de animales y personas y apenas incluye órganos internos o partes constitutivas de objetos (Moyna 2011: 88) ${ }^{2}$, pero esta limitación no está presente en los mismos compuestos en los dialectos del sardo o en los adjetivos posesivos derivados en -udo (los contrastes entre lanudo vs. *lanifino y sañudo vs. *sañirojo ejemplifican que los derivados aceptan la atribución de la posesión de lana y saña, no así el compuesto).

Nuevamente, vemos que el contraste con el otro esquema compositivo, el de (4), es evidente: la foto no es una parte integral del elemento que es sensible a ella, ni el ácido lo es de los materiales que lo resisten, ni The Beatles lo es de sus seguidores. Como en el caso de (2), la contribución semántica de los modificadores a los adjetivos nucleares no depende de sus rasgos semánticos característicos (p. e., si lo cualifican como parte de un todo o no), sino que su contribución es la de indicar a qué se es sensible, resistente o de qué se tiene miedo).

A falta de un análisis más minucioso y un mayor número de ejemplos, puesto que nos centraremos en el caso de los compuestos verbales, invitamos a reflexionar al lector sobre los contrastes de (1-4), que parecen apuntar a la existencia de ciertas correspondencias entre esquemas compositivos $(\mathrm{N}+\mathrm{N}$ y $\mathrm{N}+\mathrm{Adj})$ con diferencias morfofonológicas explícitas (número de acentos y posición del núcleo en el primer caso; selección de alomorfos en el segundo) y propiedades semánticas distintivas.

\section{ASUNCIONES TEÓRICAS DE LA PROPUESTA}

En la introducción hemos hecho referencia a la posible asignación a los sustantivos en el interior de compuestos de roles temáticos de «argumentos» $\mathrm{y}$ "adjuntos». Dado que esta idea se desarrolla en las siguientes páginas, queremos precisar que la presente contribución asume una aproximación «sintacticista» a la estructura argumental de los compuestos, aceptándose que esta se construye en la sintaxis y no viene predeterminada por el léxico. Presentaremos una visión simplificada del modelo de Ramchand (2008) para vertebrar nuestro análisis, en tanto en cuanto haremos referencia a tres posibles componentes de los eventos verbales (inicio, proceso y resultado), cada uno de los cuales introduce sus argumentos respectivos: el primero introduce como su argumento al iniciador del evento; el segundo, al paciente de un proceso; y el tercero, al participante tal y como queda tras el proceso, es decir, en David se mudó a La Laguna, David es el paciente de proceso y también su iniciador puesto que se muda y no «lo mudan»; y David, ya en La Laguna, es el argumento del estado resultante. La Laguna es un tipo de participante que en el modelo de Ramchand recibe el nombre de rema. Los remas únicamente precisan la información acerca del evento sin verse implicados en él o experimentar cambio alguno, a diferencia de David. Ejemplos habituales de este tipo de participantes son

\footnotetext{
2 Excepciones son ventripotente y sanguinemixto (con órgano interno) o puntiagudo (con parte de objeto).
} 
los complementos de verbos de duración y de medida, como dos horas en La película duró dos horas o dos metros en La tela mide dos metros.

Este modelo nos interesa porque no equipara la condición (sintáctica) de argumento de alguno de los cuatro tipos mencionados con ciertas distinciones entre roles temáticos que no tienen repercusión sintáctica, al menos en nuestro objeto de estudio, aunque sí determinan ciertas preferencias, como veremos. Así, el concepto de Iniciador puede englobar roles temáticos como los de agente (David se mudó) y diferentes tipos de iniciadores no-agentivos como causas (El ruido nos hizo marcharnos), medios (La gripe enfermó a media isla), fuerzas de la naturaleza (El volcán arrasó la ciudad).

\section{LA SEMÁNTICA DE COMPUESTOS VERBALES Y DEVERBALES}

Empezamos la discusión por aquel esquema compositivo cuyos aspectos semánticos han sido estudiados en mayor profundidad: los compuestos de verbo y nombre del tipo de lavavajillas.

Atendiendo a la relación entre compuesto y referente, el estudio de referencia es el de Bustos (1986: 274 y ss.), quien distingue entre aquellas formaciones que ponen nombre a entidades inanimadas (abrelatas) y animadas (limpiabotas). Dentro del grupo animado, distingue a su vez entre la referencia a personas (guardaespaldas), animales (quebrantahuesos) y plantas (atrapamoscas), y, dentro del grupo de los conceptos-persona en particular, entre aquellos que caracterizan al individuo de manera denotativa (recogepelotas) y los que lo hacen de manera connotativa (matasanos). A su vez, en estos últimos, se analiza la manera en que las acciones crean el efecto humorístico: por lo hiperbólico (vuelcatrenes), por lo violento (atizacandiles), por lo soez (meapilas), etc.

No obstante, no es la relación entre el compuesto en su totalidad y el referente la que nos permite caracterizar las propiedades distintivas de los sustantivos anteriores. Al fin y al cabo, los $\mathrm{N}+\mathrm{N}$ también hacían referencia a entidades animadas, como pez globo, o inanimadas, como camión cisterna. Sin embargo, el contraste entre ambos compuestos respecto a la naturaleza verbal o nominal del primer constituyente sí determina diferencias significativas en cómo los compuestos se relacionan con sus referentes.

Así, los compuestos del tipo de pez globo hacen referencia a entidades animadas e inanimadas, pero no los conceptualizan como agentes o instrumentos, mientras que el verbonominal lavavajillas sí lo hace: compárense mesa camilla y reposabrazos; del primero diríamos que denota una clase de mueble, pero no un instrumento concebido para un fin específico; sí entendemos reposabrazos de este modo. Lo mismo sucede si comparamos mujer objeto y calientabraguetas: ambas estructuras aluden peyorativamente a mujeres, pero mientras que en el verbonominal calientabraguetas la razón de condena es lo que hacen (agentes), en mujer objeto la causa es su cosificación mediante el uso del sustantivo objeto. La explicación reside en la estructura interna del compuesto: es la presencia de una proyección verbal en los compuestos 
(verbo)nominales la que hace que el potencial denotativo del compuesto incluya iniciadores de acciones como agentes e instrumentos (Varela 1990).

Desde el punto de vista de su estructura argumental, es habitual caracterizar los compuestos como lavavajillas como estructuras transitivas (el sujeto que lava y lo que se lava). Val Álvaro (1999: 4794-ss.), al igual que Scalise, Fábregas y Forza (2009), cuestiona este vínculo y ejemplifica la necesidad de dar cabida a construcciones cuyos sujetos aparentemente transcienden el esquema agente-tema. Analizaremos varios de estos ejemplos, pero no coincidimos con los autores mencionados en la necesidad de cuestionar el vínculo entre el esquema de lavavajillas y la transitividad, particularmente cuando este esquema se compara con el de los compuestos formados por un sustantivo deverbal (teniente) o adjetivo/participio (entendido) y un complemento o modificador (droga, mal), construcciones que se conocen como compuestos sintéticos o deverbales. Los ejemplos en (5) muestran cómo estos últimos se forman con naturalidad con verbos intransitivos, a diferencia del compuesto verbonominal.

5. terrateniente ( ${ }^{*}$ tienetierra), drogodependiente $\left({ }^{*}\right.$ dependedroga), cariacontecido ( ${ }^{*}$ acontececara), alicaído ( ${ }^{*}$ caeala), cabizcaído ( ${ }^{*}$ caecabeza); malcasada ( ${ }^{*}$ casamal), malentendido (*entiendemal), maldito (\#dicemal), malsonante (* ${ }^{*}$ suenamal), malherido (\#hieremal)

Los equivalentes verbonominales de los sustantivos y adjetivos deverbales de (5) son agramaticales (marcamos aquellos que podrían existir con \#). Vemos que el compuesto verbonominal no tolera verbos estativos como tener o resistir ${ }^{3}$, intransitivos como caer, acontecer o sonar, es decir, aquellos que no requieren de iniciadores de acciones y actividades. Entre los verbos intransitivos conocidos como inergativos, aquellos que presentan iniciadores se documentan ocasionalmente, como lloraduelos 'persona que frecuentemente lamenta sus infortunios'.

Varela y Felíu (2003), entre otros autores, han relacionado los contrastes anteriores con el hecho de que el esquema verbonominal presenta un nominalizador nulo que corresponde con un sufijo agentivo - dor. Esta equivalencia implicaría que los verbonominales presentan restricciones en su esquema argumental equiparables únicamente a las de formaciones como vasodilatador y no a las no agentivas como terrateniente o malherido. Como veremos a continuación, para caracterizar el potencial denotativo del compuesto verbonominal de una manera más apropiada es necesario prescindir del concepto de "agentivo" y usar en su lugar el concepto de Iniciador, puesto que nos permitirá capturar ciertas particularidades del compuesto verbonominal respecto de las construcciones deverbales.

${ }^{3}$ Como apunta un revisor, existen compuestos verbales como sabelotodo y tentetieso, que no presentan las restricciones de los verbos señaladas. Este hecho, junto con la presencia habitual de pronombres y de temas verbales diferentes al de tercera persona, refuerza la visión, mantenida por varios autores, de que estas construcciones no forman parte de la pauta productiva representada por lavavajillas, sino que son el producto de la lexicalización puntual de sintagmas. 
El contraste de lavavajillas con las construcciones de (5) se explicaría, en el marco teórico que hemos asumido, de la manera siguiente: el verbonominal lavavajillas solo presenta dos tipos de argumentos: iniciadores (lo que efectúa la acción de lavar) y remas de proceso (descripción de aquello que se lava -vajillas-, que en la estructura del compuesto nunca se lleva a cabo, stricto sensu, por lo que vajillas no es un argumento de proceso). Al no haber argumentos de proceso lógicamente no los hay de resultado (si nada experimenta un cambio no puede quedar en un estado diferente al original). Todas las versiones del esquema lavavajillas de construcciones sin iniciadores (drogodependiente, cariacontecido, alicaido) son imposibles, porque iniciador es una proyección obligatoria del verbonominal.

En contrapartida, las construcciones de (5) sí pueden incluir, a diferencia de las verbonominales, argumentos de Resultado. Por ello estas pueden denotar el estado resultante de un individuo tras eventos como sorprenderse o entristecerse -Juan (acabó) boquiabierto-cabizbajo-, o una evaluación subjetiva sobre el estado del participante una vez que eventos como casarse o ser herido ha concluido-María (acabó) malcasada-malherida.

La conclusión que puede obtenerse de las diferencias semánticas entre la estructura del esquema compositivo $\mathrm{V}+\mathrm{N}$ que representa lavavajillas y las construcciones $\mathrm{Adv} / \mathrm{N}+\mathrm{V}+$ sufijo, que representan indistintamente vasodilatador, terrateniente y malentendido es la siguiente: el potencial denotativo del compuesto verbonominal (o el conjunto de realidades extralingüísticas que pueden conceptualizar) es, definitivamente, inferior que el de las construcciones con sufijos, y la razón es que la estructura argumental del primero es rígida-Iniciadores+Remas- mientras que en el caso de las segundas puede contener todo tipo de argumentos (iniciadores, pacientes afectados por procesos, aquellos a los que se les atribuye un estado resultante de cambio...), precisamente porque la participación de sufijos activos (-dor) participiales $(-d o)$, disposicionales (-nte) modula las estructuras argumentales de las construcciones de (5) - p. e., lógicamente, en presencia de un sufijo pasivo no va a haber iniciadores agentivos-. El compuesto verbonominal no presenta sufijo alguno (Marqueta 2018), y, por ende, su potencial denotativo es mucho menor.

A continuación, retomamos la idea de por qué hacer referencia al compuesto verbonominal lavavajillas como agentivo/instrumental es una explicación aceptable pero simplificadora de su potencial denotativo. Recordamos que, de acuerdo con nuestra hipótesis, dicho potencial no se explica por los valores de un sufijo nominalizador equivalente a -dor $\mathrm{u}$ otros sufijos similares, sino por los diferentes roles temáticos que pueden asignarse a los argumentos iniciadores y a los argumentos remas. En línea con lo que vimos en páginas anteriores, veremos que la interpretación semántica de dichos roles temáticos varía en función del tipo de evento al que el constituyente verbal hace referencia. 


\section{A) Verbos que dan lugar a 'Instrumentos' y 'Agentes'}

Oscilamos entre casos claros como abrelatas y elevalunas, donde la entidad inanimada denotada tiene capacidad de iniciar la acción gracias a su mecanismo o motor, hasta casos donde la entidad denotada, por ejemplo, una pipeta en catalicores, carece de tal mecanismo, de modo que el iniciador del evento será, por extensión, el usuario que lo manipula. En construcciones como pasamontañas, portamonedas, reposacabezas y cuelgacapas la entidad inanimada denotada parece ser un participante secundario de la acción: es una entidad animada o agente la que, stricto sensu, 'pasa la montańa', 'porta las monedas' y 'cuelga la capa' y el compuesto expresa con qué lo hace ${ }^{4}$.

Notamos que en las parejas agentivo-instrumentales la interpretación de entidades animadas favorece la literalidad de la acción (6):

\section{6. salvapatrias/salvamanteles, guardaespaldas/guardarropa, rompetechos/rompeolas}

Lo cual no quiere decir que las entidades animadas no adquieran tintes metafóricos (un cazafortunas no caza). Esto sucede particularmente cuando el verbo denota originariamente acciones exageradas, violentas o escatológicas (tragaldabas, atropellaplatos, desuellacaras, azotacalles, ahogaviejas, ahorcaperros, destripaterrones, lameculos, chupapostes).

\section{B) Verbos QUe PRESENTAN TIPOS DE INICIADORES AUSENTES EN LOS DERIVADOS EN $-D O R$}

Los derivados en -dor denotan iniciadores agentes (colonizador), instrumentos (abridor) y medios (fertilizador). Todos ellos también abundan en el verbonominal.

Son medios comunes para los derivados en -dor y los compuestos verbonominales los cosméticos como quitaesmalte (crema purificadora, reparadora...). Hay, sin embargo, ciertas clases que le son propias al esquema verbonominal: los 'accidentes'-geográficos o de diversa naturaleza-, que responden al esquema causa-efecto sin tener propiedades de instrumentos cuya acción sea manipulable por parte de agentes -(puerto de) arrebatacapas, cortafuegos, rompeolas, quitamiedos-y las fuerzas de la naturaleza como el viento (rabiazorras) o la lluvia (calabobos).

${ }^{4}$ Dejamos pendiente para un análisis posterior la explicación de cómo se establece la correspondencia, en estos casos entre el Iniciador, en tanto que componente estructural obligatorio del compuesto, y el iniciador conceptual o «real» de la acción que se denota, particularmente en los casos como catalicores o cuelgacapas. En esos dos ejemplos (la pipeta y el mueble por su usuario), resulta plausible que la metonimia sea de alguna manera un factor clave para garantizar la interpretabilidad. 
Puede comprobarse que rompedor y arrebatador no tienen nada que ver con la iniciación de eventos (son cualidades), quitador y calador no se usan y ${ }^{*}$ rabiador está mal formado.

Cabe matizar que lo contrario también sucede, a saber: iniciadores posibles en los derivados en -dor y que no están atestiguados en los compuestos verbonominales: soñador/\#sueñamundos, madrugador/\#madrugalunes; vividor/*vivevidas; predicador/\#predicabiblias; dictador/*dictaórdenes; hablador/\#hablalatín.

\section{C) Verbos Con iniciadores causativos (No Agentivos)}

En el verbonominal podemos identificar claramente compuestos causativos desvinculados de la agentividad (7), a los que podemos considerar verbos causativizados por la estructura argumental biargumental (Inicio-rema) del compuesto.

7. a. andaniños, crecepelo, tardanaos, rabiazorras, ardeviejas, resbalabueyes, saltatumbas b. engañabobos, amargacenas, espantapájaros

Todos los ejemplos de (7a) se interpretan de manera causativa, aunque se forman con verbos no causativos. Andaniños designa a un artefacto que no anda, sino que hace que los niños anden; crecepelo, un producto que hace que el pelo crezca; tardanaos, un pez que retrasa los barcos; rabiazorras, un viento que "hace rabiar" a las zorras; ardeviejas, una planta (espinosa) que, entendemos, quema a las viejas; una planta es también resbalabueyes (Moyna 2011: 409). Finalmente, en el español de Aragón, a un tobogán se le llama esbarizaculos 'resbalaculos' y el verbo de actividad saltar se emplea como su versión causativa/de acción asaltar en saltatumbas 'cura gorrón'. Todos los verbos psicológicos con alternancia sujeto/objeto experimentante se admiten únicamente en la lectura con sujeto causativo (7b).

Todas estas construcciones ponen en evidencia que la estructura argumental del compuesto no viene siempre determinada por el verbo de base, puesto que es el compuesto el que impone su propio perfil argumental (Iniciador-Rema) a los verbos y nombres que en él aparecen. Un iniciador que no es necesariamente un agente.

\section{D) Verbos rematizados}

Mientras el esquema verbonominal es especialmente reacio a admitir verbos de movimiento que implican trayectoria ( ${ }^{*}$ entrasillas, salecasas, *avanzametros, ${ }^{*}$ subemontañas, *bajacuestas, *llegapueblos, *acudemisa, *huyeciudades, *vaextranjero-, sí presenta muchos verbos de manera de movimiento (8a). La razón es que estos pueden confluir en la misma estructura argumental que los verbos de actividad (8b); por ejemplo, añadiendo a su estructura argumental habitual intransitiva un argumento «rema» (vagar el mundo, saltar los montes, correr las calles). 
8. a. vagamundos, trotaconventos, saltamontes, arrastrapanza, correcalles, vuelvepiedras; tornapeón, torcecuello, rodapié, rodeabrazo

b. mondadientes, lavavajillas, friegasuelos, limpiacristales, secamanos

Los argumentos remas del compuesto verbonominal están semánticamente restringidos desde el punto de vista de las relaciones entre constituyentes, puesto que necesariamente ha de interpretarse con roles temáticos asociados típicos de los argumentos internos ${ }^{5}$. Sin embargo, los sustantivos de los compuestos deverbales, al igual que vimos para el caso de los $\mathrm{N}+\mathrm{N}$ como hidroavión, pueden dar cabida a diferentes tipos de argumentos y adjuntos, no solo los mismos que el VN farmacovigilancia 'que vigila los efectos de fármacos', sino también adjuntos como locaciones (eurodirigente), vías (cableoperador), agentes (autoadministrado), etc.

\section{CONCLUSIONES}

A lo largo de este trabajo, hemos analizado algunos aspectos de la semántica de los compuestos con verbos en español que parecen condicionados por su estructura. Entre dichos aspectos, hemos encontrado, conforme a la expectativa, aquellos que tradicionalmente forman parte de la semántica estructural, como la presencia de roles temáticos asociados a la (in)transitividad del esquema, pero también algunos que, típicamente, se asocian a las idiosincrasias de la entrada léxica del verbo, como el tipo de referente denotado. El objetivo con este análisis, aún incipiente, es llamar la atención acerca de la presencia de una mayor sistematicidad en la vertiente semántica de la composición de la que normalmente se considera. Una posible extensión de la propuesta es la búsqueda de sistematicidades similares en otras clases de compuestos del español y/o procedimientos de formación de palabras.

ReCibido: mayo de 2019; ACEPTADo: octubre de 2019.

5 Hablamos de roles temáticos como el de paciente si la entidad es animada (espantapájaros), o el homónimo tema para entidades inanimadas (lavaplatos) y abstractas (quitamiedos), pero no de roles temáticos que nunca se codifican en dicha posición, como destinatario ( ${ }^{*}$ escribeprimas) o meta (*llegaciudades). El rol temático de locación (correcaminos, pasacalles) tiene cabida dentro del concepto de tema incremental. 


\section{BIBLIOGRAFÍA}

Acкema, Peter y Ad Neeleman (2004): Beyond Morphology. Interface Conditions on Word Formation, Oxford: Oxford University Press.

Bauer, Laurie (2008): «Exocentric compounds», Morphology 18(1): 51-74

Beard, Robert (1995): Lexeme-Morpheme Base Morphology. A General Theory of Inflection and Word Formation, New York: State University of New York Press.

Buenafuentes de la Mata, Cristina (2017): «Aportaciones de la semántica cognitiva a la formación de palabras en composición», RILCE 33(4): 1063-1090.

Bustos, Eugenio (1986): La composición nominal en español, Salamanca: Universidad de Salamanca.

Downing, Pamela (1977): «On the creation and use of English compound nouns», Language 53(4): 810-842.

FÁbregas, Antonio (2005): La definición de la categoría gramatical en una morfología orientada sintácticamente. Nombres y adjetivos, tesis doctoral, Madrid: Universidad Autónoma de Madrid.

GaGnÉ, Cristina y Thomas Spalding (2006): «Conceptual Combination. Implications for the Mental Lexicon», en Gary Libben y Gonia Jarema (eds.), The Representation and Processing of Compound Words, New York: Oxford University Press, 145-168.

GIL LAFORGA, Irene (2014): La interacción de los componentes gramaticales en la formación de palabras: adjetivos posesivos derivados y compuestos, tesis doctoral, Madrid: Universidad Autónoma de Madrid.

JACKendoff, Ray (2016): «English NN compounds in Conceptual Semantic», en Pius ten Hacken (ed.), The Semantics of Compounding, Cambridge: CUP, 15-37.

Lees, Robert (1960): The Grammar of English Nominalizations, The Hague: Mouton.

Lieber, Rochelle (2004): Morphology and Lexical Semantics, Cambridge: CUP.

Lieber, Rochelle (2016): English Nouns. The Ecology of Nominalization, Cambridge: CUP.

Marqueta, Bárbara (2018): «Una propuesta de categorización sin morfemas para el compuesto lavaplatos», Estudios de Lingüistica de la Universidad de Alicante 32: 219-236.

Moyna, Irene (2011): Compound Words in Spanish. Theory and history, Amsterdam: John Benjamins.

PLAG, Ingo (1999): Morphological productivity: Structural constraints in English derivation, Berlin: Mouton.

Pustejovsky, James (1995): The Generative Lexicon. A Theory of Computational Lexical Semantics, Cambridge: MIT Press.

Pinto, Immacolata, Giulio Paulis e Ignazio Putzu (2012): «Sardinian adjectives with the NiA structure», Lingue e Linguaggio 11(1): 49-70.

Rainer, Franz y Soledad Varela (1992): «Compounding in Spanish», Rivista di Lingüistica 4(1): 117-142.

Ramchand, Gillian (2008): Verb Meaning and the Lexicon. A First Phase Syntax, Cambridge: CUP.

Scalise, Sergio, Antonio Fábregas y Francesca Forza (2009): «Exocentricity in Compounding», Gengo Kenkyū 135: 49-84.

Tump, Gregory (2001): Inflectional Morphology: A Theory of Paradigm Structure, Cambridge: CUP. 
Val Alvaro, José Francisco (1999): «La composición», en Ignacio Bosque y Violeta Demonte (eds.), Gramática Descriptiva de la Lengua Española, Madrid: Espasa-Calpe, 4757-4841.

VArela, Soledad y Elena Felíu (2003): «Internally motivated structural borrowing in Spanish morphology», en Paula Kempchinsky y Carlos Piñeros (eds.), Theory, Practice and Acquisition, Sommerville, MA: Cascadilla Press, 83-101. 
